Pacific Journal of Mathematics

ENGELS THEOREM FOR A CLASS OF ALGEBRAS 


\title{
ENGEL'S THEOREM FOR A CLASS OF ALGEBRAS
}

\author{
ERNEST L. STITZINGER
}

A condition of nilpotency is derived for a class of algebras which include the almost alternative algebras of A. A. Albert. This result is seen to be an extension of Engel's theorem. Some consequences are then considered.

An algebra $A$ over a Noetherian ring $R$ is called almost alternative if it is power associative and satisfies the following identities

I.

$$
\begin{aligned}
u(v w)= & \alpha_{1}(u v) w+\alpha_{2} w(u v)+\alpha_{3}(v u) w \\
& +\alpha_{4} w(v u)+\alpha_{5}(u w) v+\alpha_{6} v(u w)+\alpha_{7}(w u) v+\alpha_{8} v(w u)
\end{aligned}
$$

and

II.

$$
\begin{aligned}
(v w) u= & \beta_{1} v(w u)+\beta_{2}(w u) v+\beta_{3} v(u w) \\
& +\beta_{4}(u w) v+\beta_{5} w(v u)+\beta_{6}(v u) w+\beta_{7} w(u v)+\beta_{8}(u v) w
\end{aligned}
$$

where $u, v, w \in A$ and $\alpha_{i}, \beta_{i} \in R$. In what follows the requirement of power associativity will not be needed. Lie, alternative and $(\gamma, \delta)$-algebras are contained in this class. It is the purpose of this note to give a criterion for nilpotency inspired by the Engel theorem in Lie algebras. However, it is not sufficient to assume that each multiplication is nilpotent. For let $A$ be a 3-dimensional algebra generated by $x, y$ and $z$ where $x y=z$ and $z x=y$ and all other multiplications between basis elements are 0 . Then $A$ is a non-nilpotent algebra satisfying I and II with $\alpha_{4}=\beta_{4}=1$ and all other $\alpha_{i}, \beta_{i}=0$. Also each right and left multiplication by any element in $A$ is nilpotent. Note that $A$ is not power associative.

Let $R$ be a Noetherian ring. All algebras and modules over $R$ are assumed to be unital. Let $A$ be an algebra over $R$ satisfying I and II. For each $x \in A, R_{x}$ and $L_{x}$ will denote right and left multiplication of $A$ by $x$. Let $M$ be an $A$-bimodule (see [4, p. 25]) with induced representation $(S, T)$. Hence $S$ and $T$ satisfy identities derived from I and II and consequently $S_{x y}, T_{x y} \in\left\langle S_{x}, S_{y}, T_{x}, T_{y}\right\rangle$, the associative subalgebra of $\operatorname{End}(M)$ generated by $S_{x}, S_{y}, T_{x}, T_{y}$. In particular, if $x \in A$ and $y$ is in the 
subalgebra of $A$ which is generated by $x$, then $S_{y}, T_{y} \in\left\langle S_{x}, T_{x}\right\rangle$. This latter subalgebra will be denoted by $J(x)$. Also recall that a subset of $A$ which is closed under multiplication is called a Lie set.

THEOREM. Let $A$ be an algebra over a Noetherian ring $R$ such that $A$ satisfies I and II. Let $M$ be an A-bimodule such that $M$ is a finitely generated $R$-module. Let $C$ be a Lie set in $A$ such that $C$ generates $A$. Suppose that for each $x \in C, J(x)$ is nilpotent. Then $A$ acts nilpotently on $M$.

Proof. There exist Lie subsets $G$ of $C$ such that the algebra generated by $G,\langle G\rangle$, acts nilpotently on $M$ since the algebra generated by any $x \in C$ has this property. This follows from the remarks preceding the statement of the theorem. Also $\{x \in A ; M x=x M=0\}$ is an ideal in $A$. Hence assume that the representation is faithful and that $A$ is finitely generated. Let $D$ be a Lie subset of $C$ such that $\langle D\rangle$ acts nilpotently on $M$ and $\langle D\rangle$ is maximal with this property. Since we may assume that $A$ contains $\langle D\rangle$ properly, $C \nsubseteq\langle D\rangle$. By assumption there exists $n$ such that $M \sigma_{1} \cdots \sigma_{n}=0$ for all possible $\sigma_{1}, \ldots, \sigma_{n}$ where $\sigma_{i}=S_{x_{1}}$ or $\sigma_{i}=T_{x_{i}}$ and each $x_{i} \in D$. Let $M_{i}=\left\{m \in M ; m \sigma_{1} \cdots \sigma_{i}=0\right.$ for all possible $\left.\sigma_{1}, \ldots, \sigma_{l}\right\}$. Then $0=M_{0} \subseteq M_{1} \subseteq \cdots \subseteq M_{n}=M$.

Now consider any element of the form $\sigma_{1} \cdots \sigma_{i-1} \tau \sigma_{l} \cdots \sigma_{2 n}$ where each $\sigma_{i}$ is as defined above, $\tau=S_{a}$ or $\tau=T_{a}$ and $i=1, \ldots, 2 n-1$. This product must be 0 . Next consider any $y \in A$ with $2 n+1$ factors of which $2 n$ are from $D$. Both $S_{y}$ and $T_{y}$ are the sum of terms of the above type, hence $S_{y}=T_{y}=0$. Hence $y=0$. Now there exists a least positive integer $m$ such that $C \tau_{1} \cdots \tau_{m} \subseteq\langle D\rangle$ for all $\tau_{1}, \ldots, \tau_{m}$ where each $\tau_{i}=R_{x_{i}}$ or $\tau_{i}=L_{x_{i}}$ and $x_{i} \in D$. Then there exists $z=x \tau_{1} \cdots \tau_{m-1} \notin\langle D\rangle$ and $x \in C$. Now $z \in C$ since $C$ is a Lie set and if $y$ is the product of $z$ 's, no matter how associated, then $y \in C$, and using I and II, $y D, D y \subseteq D$. If $F$ is the union of $D$ and all such $y$, then $F$ is a Lie subset of $C$ and $\langle F\rangle$ contains $\langle D\rangle$ properly.

It remains only to show that $\langle F\rangle$ acts nilpotently on $M$. Under the conditions on the representation, $S_{z}$ and $T_{z}$ leave each $M_{i}$ invariant and $J(z)$ acts nilpotently on each factor. Furthermore $S_{y}, T_{y} \in J(z)$ where $y$ is any product of $z$ 's. Then the chain may be refined to one such that $F$, and hence $\langle F\rangle$, annihilates each factor. This contradiction establishes the result.

The following are extensions of results on Lie algebras to the present setting. 
Corollary 1. Let $A$ and $R$ be as in the theorem and let $N$ be a nilpotent ideal of $A$. Let $U(N, A)$ be the associative subalgebra of $\operatorname{End}(A)$ generated by $R_{x}, L_{x}$ for all $x \in N$. Then $U(N, A)$ is contained in the radical of $U(A, A)$.

Proof. Since $N$ is a nilpotent ideal of $A, J(n)$ is nilpotent for each $n \in N$. Hence $U(N, A)$ is nilpotent by the theorem. Hence these exists a chain $0=A_{0} \subseteq \cdots \subseteq A_{k}=A$ where $A_{j}=\left\{x \in A ; N x+x N \subseteq A_{J-1}\right\}$. Clearly $A A_{j}+A_{j} A \subseteq A_{j}$. Let $\sigma=\sigma_{1} \cdots \sigma_{n}$ where $\sigma_{i}=S_{x_{i}}$ or $\sigma_{l}=T_{x_{i}}$, $x_{i} \in A$, and at least one $x_{i} \in N$. Then $T$ annihilates each factor in the chain. Hence the ideal of $U(A, A)$ generated by $U(N, A)$ has nilpotent length $k$.

\section{Using the regular representation, the theorem becomes}

COROllary 2. Let $A$ be finitely generated over the Noetherian ring $R$. Suppose that $A$ satisfies I and II. Let $C$ be a Lie set in $A$ such that $C$ generates $A$. If $J(x)$ is nilpotent for all $x \in C$, then $A$ is nilpotent.

The next application deals with the nilpotency of algebras which admit regular automorphisms. This is an extension of a Lie algebra result of Jacobson [3]. Note that algebras satisfying I and II remain in this class under extension of the base.

COROllary 3. Let $A$ be a finite dimensional algebra over a field. Suppose that $A$ satisfies I and II. Let $\Phi$ be an automorphism of $A$ such that $\Phi^{p}=I$ where $p$ is a prime. Suppose that $\Phi$ has no non-zero fixed points. Then $A$ is nilpotent.

Proof. We may assume that the base is algebraically closed. Let $\alpha_{1}, \ldots, \alpha_{n}$ be the roots of $\Phi$, all of which are $p$-roots of unity other than 1, and let $A=A_{\alpha_{1}} \oplus \cdots \oplus A_{\alpha_{n}}$ be the decomposition of $A$ into characteristic subspaces. For roots $\alpha, \beta, A_{\alpha} A_{\beta} \subseteq A_{\alpha \beta}$. Hence $C=U A_{\alpha_{t}}$ is a Lie set in $A$. Let $x \in A_{\alpha}$ and $\beta$ be any $p$-root of unity. Then there exists $i, 1 \leq i \leq p$, such that $\beta \alpha^{i}=1$. If $\sigma$ is the product of $i$ terms, each of which is $R_{x}$ or $L_{x}$, then $A_{\beta} \sigma \subseteq A_{1}=0$. Hence if $\sigma$ is the product of $p$ or more such terms, then $A_{\gamma} \sigma=0$ for all roots $\gamma$. Hence $A \sigma=0$. Now the product of $p$ elements from $J(x)$ can be expressed as the sum of terms each with at least $p$ factors all of the form $R_{x}$ or $L_{x}$. Hence $J(x)^{p}=0$. Therefore $A$ is nilpotent. 
Again let $A$ satisfy I and II and for $x \in A$, let $E_{A}(x)=\{y \in$ $A ; y J(x)^{k}=0$ for some $\left.k=1,2, \ldots\right\}$. Then

LeMma. $E_{A}(x)$ is a subalgebra of $A$.

The Frattini subalgebra $\phi(A)$ of an arbitrary algebra $A$ has been investigated by Towers [5]. In this direction we obtain a generalization of a theorem of D. W. Barnes.

COROllaRy 4. Let $A$ be a finite dimensional algebra over a field. Suppose that $A$ satisfies I, II and is flexible. Let $N$ be an ideal of $A$ such that $N \subseteq \phi(A)$ and $A / N$ is nilpotent. Then $A$ is nilpotent.

Proof. Suppose not. Then there exists $x \in A$ such that $J(x)$ is not nilpotent. For $\sigma \in J(x)$ let $A_{0 \sigma}$ and $A_{1 \sigma}$ be the Fitting null and one spaces of $A$ with respect to $\sigma$. Since $A$ is flexible, $J(x)$ is abelian and $A_{0 \sigma}$ and $A_{1 \sigma}$ are $\tau$-invariant for each $\tau \in J(x)$. Since $A / N$ is nilpotent, $A_{1 \sigma} \subseteq N$ for each $\sigma$, hence $B=\bigcap A_{0 \sigma}$ supplements $N$. Also $E_{A}(x) \subseteq B$. Now $J(x)$ restricted to $B$ is a nilalgebra, hence it is nilpotent. Hence $E_{A}(x)=B$. Then $A=E_{A}(x)+N=E_{A}(x)+\phi(A)$, a contradiction since $E_{A}(x)$ is a subalgebra of $A$.

The final result concerns the existence of Cartan subalgebras, nilpotent self-normalizing subalgebras. Clearly these can not exist in general. However, we have the following result.

Corollary 5. Let $A$ be a finite dimensional algebra over a field. Suppose that $A$ is solvable, flexible and satisfies I and II. Then $A$ contains $a$ Cartan subalgebra.

Proof. Induct on the dimension of $A$. Let $M$ be minimal ideal of $A$. There exists $B \subseteq A$ such that $B \supseteq M$ and $B / M$ is a Cartan subalgebra of $A / M$. If $B \neq A$, then there exists a Cartan subalgebra $C$ of $B$. $C$ is nilpotent and we claim that $C$ is self-normalizing in $A$. Let $D=N_{A}(C)$, the normalizer of $C$ in $A$. Since $C+M / M$ is a Cartan subalgebra of $B / M, C+M=B$. If $x \in D$, then $x \in N_{A}(B)=B$. Hence $D \subseteq B$. Therefore $D=N_{B}(C)=C$ and $C$ is a Cartan subalgebra of $A$.

Suppose $B=A$. Then $A / M$ is nilpotent and we may assume that $A$ is not nilpotent. By the above corollary, $M \nsubseteq \phi(A)$. Hence $A$ contains a maximal subalgebra $T$ which complements $M$ in $A$ since $T \cap M$ is an ideal 
in $T+M=A . T$ is nilpotent and $N_{A}(T) \cap M$ is an ideal in $N_{A}(T)+$ $M=A$. Then either $N_{A}(T) \cap M=0, N_{A}(T)=T$ and $T$ is a Cartan subalgebra of $A$ or $N_{A}(T) \cap M=M$. Then $T$ is an ideal in $A$ and $A=A / T \cap M$ is nilpotent, a contradiction.

\section{REFERENCES}

[1] A. A. Albert, Almost alternative algebras, Portugals Math., 8 (1949), 23-26.

[2] D. W. Barnes, On the cohomology of soluble Lie algebras, Math. Z., 101 (1967), 343-349.

[3] N. Jacobson, $A$ note on automorphism and derivations of Lie algebras, Proc. Amer. Math. Soc., 6 (1955), 281-283.

[4] R. D. Schafer, An Introduction to Non-associative Algebras, Academic Press, New York, 1966.

[5] D. A. Towers, A Frattini theory for algebras, Proc. London Math. Soc., (3) 2 (1973), 440-462.

Received August 4, 1981.

North Carolina State University

RALEIGH, NC 27650 



\section{PACIFIC JOURNAL OF MATHEMATICS}

\section{EDITORS}

Donald BabbitT (Managing Editor)

University of California

Los Angeles, CA 90024

Hugo Rossi

University of Utah

Salt Lake City, UT 84112

C. C. Moore and Arthur Ogus

University of California

Berkeley, CA 94720
J. DugunduI

Department of Mathematics

University of Southern California

Los Angeles, CA 90089-1113

R. FinN and H. SAmelson

Stanford University

Stanford, CA 94305

\section{ASSOCIATE EDITORS}
R. ARENS
E. F. BECKENBACH
B. H. NEUMANN
F. WOLF
K. YosHIDA (1906-1982)

\section{SUPPORTING INSTITUTIONS}

UNIVERSITY OF ARIZONA

UNIVERSITY OF BRITISH COLUMBIA

CALIFORNIA INSTITUTE OF TECHNOLOGY

UNIVERSITY OF CALIFORNIA

MONTANA STATE UNIVERSITY

UNIVERSITY OF NEVADA, RENO

NEW MEXICO STATE UNIVERSITY

OREGON STATE UNIVERSITY
UNIVERSITY OF OREGON

UNIVERSITY OF SOUTHERN CALIFORNIA

STANFORD UNIVERSITY

UNIVERSITY OF HAWAII

UNIVERSITY OF TOKYO

UNIVERSITY OF UTAH

WASHINGTON STATE UNIVERSITY

UNIVERSITY OF WASHINGTON 


\section{Pacific Journal of Mathematics}

Vol. 107, No. $1 \quad$ January, 1983

John Kelly Beem and Phillip E. Parker, Klein-Gordon solvability and the

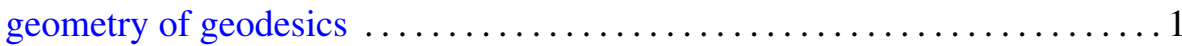

David Borwein and Amnon Jakimovski, Transformations of certain

sequences of random variables by generalized Hausdorff matrices ..... 15

Willy Brandal and Erol Barbut, Localizations of torsion theories . . . . . . . 227

John David Brillhart, Paul Erdős and Richard Patrick Morton, On sums

of Rudin-Shapiro coefficients. II ........................... 39

Martin Lloyd Brown, A note on tamely ramified extensions of rings $\ldots \ldots \ldots 71$

Chang P'ao Ch'ên, A generalization of the Gleason-Kahane-Żelazko

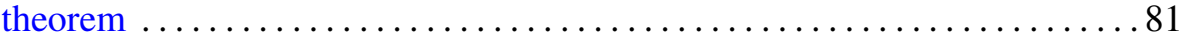

I. P. de Guzman, Annihilator alternative algebras $\ldots \ldots \ldots \ldots \ldots \ldots$. . . . 89

Ralph Jay De Laubenfels, Extensions of $d / d x$ that generate uniformly

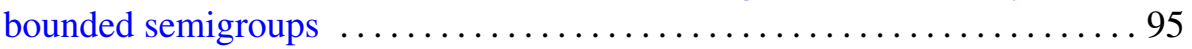

Patrick Ronald Halpin, Some Poincaré series related to identities of $2 \times 2$

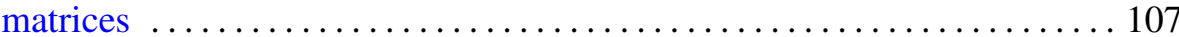

Fumio Hiai, Masanori Ohya and Makoto Tsukada, Sufficiency and

relative entropy in $*$-algebras with applications in quantum systems . . . 117

Dean Robert Hickerson, Splittings of finite groups $\ldots \ldots \ldots \ldots \ldots \ldots \ldots 14$

Jon Lee Johnson, Integral closure and generalized transforms in graded

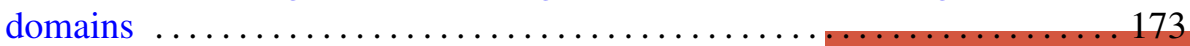

Maria Grazia Marinari, Francesco Odetti and Mario Raimondo, Affine

curves over an algebraically nonclosed field . ................. 179

Douglas Shelby Meadows, Explicit PL self-knottings and the structure of

PL homotopy complex projective spaces $\ldots \ldots \ldots \ldots \ldots \ldots \ldots \ldots \ldots$

Charles Kimbrough Megibben, III, Crawley's problem on the unique

$\omega$-elongation of $p$-groups is undecidable .................... 205

Mary Elizabeth Schaps, Versal determinantal deformations $\ldots \ldots \ldots \ldots 213$

Stephen Scheinberg, Gauthier's localization theorem on meromorphic

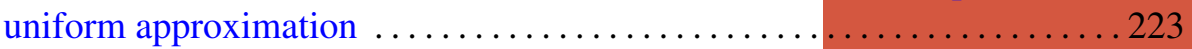

Peter Frederick Stiller, On the uniformization of certain curves . . . . . . . 229

Ernest Lester Stitzinger, Engel's theorem for a class of algebras . . . . . . . . 245

Emery Thomas, On the zeta function for function fields over $F_{p} \ldots \ldots \ldots 251$ 